\title{
OBJECT-ORIENTED SIMULATION OF PARATROOPER-VORTEX INTERACTIONS
}

\author{
T. Glenn Bailey \\ Jose C. Belano III \\ Philip S. Beran \\ Jack M. Klorber Jr. \\ Hans J. Petry \\ Department of Operational Sciences \\ Air Force Institute of Technology \\ 2950 P Street, Bldg. 640 (AFIT/ENS) \\ Wright-Patterson AFB, OH 45433-7765, U.S.A.
}

\begin{abstract}
The C-17 Airdrop Model provides the Air Force and Army test and evaluation community with the capability to (i) assess the risk of vortex encounters, and (ii) predict ground dispersal patterns on the drop zone. Written in MODSIM III, aircraft vortices and paratroopers are modeled as objects whose behavior accurately reflects their real-world counterparts through the use of pre-validated aerodynamic and trajectory equations within the objects' methods. This model has provided the joint airborne community with a preliminary estimate of paratrooper/vortex encounter rates and locations as a function of formation element separation and wind conditions, as well as a tool for simulating alternative C-17 aircraft formation configurations prior to actual flight. Future use of the model suggests simulating brigade-size operations; expanding to include equipment drops, new parachute deployment systems, and precision airdrop; visualization; and, evaluating new airborne combat tactics.
\end{abstract}

\section{INTRODUCTION}

During operational testing of airborne activities with the C-17 in June 1995, a severe encounter between a paratrooper and a vortex caused by a preceding aircraft prompted the re-examination of the formation geometry (Blake 1996). This re-evaluation confirmed that the size, weight, and aerodynamics of the $\mathrm{C}-17$ produces a wingtip vortex strength significantly greater than those previously encountered using more recent airdrop platforms of C-130s and C141s (Natick 1996b). Although the vortices dissipate over time, the strength of those produced by the C-17 poses a greater risk of severe encounters with parachute-payload systems in formation air drops. Consequently, when interacting with a parachutepayload system, these vortices can induce potentially hazardous conditions to include parachute collapse, partial deflation, severe oscillation, increased rate of descent, collision, entanglement, and hard landings (Johnson 1988, Johnson and Reynolds 1988).

Since the cost of testing and evaluating new formation tactics is high, the need for a modeling tool which can assess the probability of encounter between paratroopers and vortices becomes essential. Additional support for such a model is found in the recommendations of the previous studies by Johnson (1988) suggesting that wake vortices should be key considerations in developing and evaluating formation tactics for mass airborne airdrop activities involving paratroopers and their equipment. Thus, we developed an object-oriented simulation model that captures the key components of this system - the C-17, its vortices, and the paratroopers - into objects that reflect their interactive, dynamic, and stochastic behavior. The results of our initial set of analyses confirm that both element lead separation distance and wind conditions significantly affect the vortex encounter rate; thus, providing a basis for combat planners to make tradeoffs between paratrooper-vortex encounters, training objectives, and combat effectiveness.

The following sections describe how this objectoriented simulation model was developed and applied. Section 2 presents the background and the model's key assumptions, while Section 3 describes its development. Section 4 presents our initial results, and Section 5 concludes with lessons learned and further model enhancements. 


\section{BACKGROUND}

The C-17 Airdrop Model essentially incorporates within its component objects the research results of two key areas - parachute-payload trajectories and aircraft vortices. In both cases, a defining characteristic of the model's component objects is converting well-defined static-deterministic models from the literature into their respective methods. The underlying assumption is that by reproducing their known behavior within each object's methods, and introducing random perturbations such as winds, weight, and aircraft position, the encounter rate of the vortex and paratrooper objects can be accurately estimated for any given formation. The following subsections briefly describe the relevant literature in these two areas.

\subsection{Parachute-Payload Trajectories}

Several prior studies have looked at paratrooper performance in the context of airborne operations. Martin and Hyper (1978) address the issue of missed distance errors experienced during Canadian Forces Paradrop Exercises through the use of an approximation method called the Computed Air Release Point (CARP) to model the impact point of the parachutepayload system. This simulation varies those input parameters considered most important - winds, position at release point, and ballistics or hesitation error - and considers only individual parachute-payload systems (not formations). Johnson (1988) focuses more on vortex encounter, rather than on DZ dispersion, with C-130, C-141, and C-5 aircraft, while Blake (1996) went further into paratrooper-wake vortex encounters in an effort to predict the relative locations of paratroopers and wake vortices of the $\mathrm{C}-17$ aircraft during formation airdrops. The Blake model uses a simple point-mass system with 3 degrees of freedom (DOF), one each for the vertical, horizontal, and lateral components of motion. The Blake model can be modified to take into account a specific formation geometry; however, it does not attempt to predict dispersion on the drop zone (DZ).

Since a review of the literature found no direct parallel to our object-oriented approach, our focus shifted to selecting the most appropriate trajectory model from the literature. This review found additional 3-DOF models, including Benney (1996a) that uses a rigid two-body model that oscillates in a twodimensional wind profile; and, Wallace (1996) that models an inherent Dispersion Error Probability for a non-gliding (unconscious) parachute-payload system. By contrast, a 6-DOF model increases the resolution of the trajectory and can account for the position components of the trajectory which are the vertical, horizontal, and lateral movements (with corresponding axes) of the parachute-payload system, as well as three added DOF for rotation about each axes. One such model, developed by Tory and Ayres (1977), uses a rigid two-body system and models the trajectory of the 6-DOF parachute-payload system after full canopy inflation. Doherr (1992) develops a more complex model having 9-DOF, which uses a non-rigid two-body system where two masses, the parachute and the payload, are connected by a joint. Additionally, there are 6-DOF models associated with the payload (position and attitude) and 3-DOF models associated with the parachute (attitude only). In a complete fluid dynamic study of parachute structural dynamics in a close surface investigation, thousands of DOF are required due to the ever-increasing complexities involved, and therefore is beyond the scope of this effort. As described in detail in Section 3, we chose to incorporate the Purvis (1987) 6-DOF model for the trajectory propagation methods of the paratrooper objects.

Finally, in communicating trajectory-modeling methods in the airborne test and evaluation community, the stages of system flight are commonly referred to as zones. Zone 1 is defined from the time of exit to canopy inflation just after first vertical (normally 130 below exit for the $\mathrm{T}-10 \mathrm{C}$ ). Zone 2 is the region from 130 feet below exit to 450 feet above ground level (AGL). In combat jumps, Zone 2 is non-existent since the paratroops exit at $500 \mathrm{AGL}$. Zone 3 is the region from $450 \mathrm{AGL}$ to ground. In combat jumps, Zone 3 is defined as the region from 130 feet below exit to ground level (Natick 1996a).

\subsection{Wingtip Vortices}

The literature reveals a limited number of prior attempts at modeling wingtip vortices in an airdrop context. MAC Project 15-105-86 (Johnson 1988) includes a computer model (written in BASIC) for predicting paratrooper-vortex interactions, but is limited by the test data available at the time. Recent advances in measuring vortex behavior through the use of laser radar (Hannon et al. 1995) allowed Blake (1996) to model vortices using an elliptical load representation of a fully rolled up vortex to calculate its strength and vertical velocity. His model represents the vortex as six data points - $\mathrm{x}, \mathrm{y}, \mathrm{z}$ coordinates; strength; radius; and age - spaced at 200 -foot intervals. We adopted a modified version of his elliptical load representation as the basis for modeling C-17 vortices. 


\section{MODEL DEVELOPMENT}

\subsection{Paratrooper Objects}

We decided to model a 6-DOF model to capture the additional information that a 6-DOF model provides over a 3-DOF model, and because a 9-DOF model would provide marginal additional benefits (Benney 1996b). The selected Purvis (1987) 6-DOF model (written in FORTRAN) is a simple, yet effective, model that provides a straight forward approach to trajectory propagation by using a second-order $\mathrm{Eu}$ ler integration scheme (Doherr 1992). The approach was to first translate the original Purvis FORTRAN model code into an object method; then modify it to reflect the aerodynamic properties of a combat equipped paratrooper using a T-10C parachute; integrate the paratrooper objects with the C-17 aircraft and wake vortex object; and, finally incorporate stochastic perturbation in the trajectory propagation scheme.

The Purvis model performs three distinct roles - parameter input specific to the parachute-payload system, initialization of starting conditions, and trajectory propagation. The following four MODSIM III modules perform these functions.

The Main Module describes the activities taking place in the simulation. In the Main Module, a new instance of a jumper object, emulating the same type of parachute-payload system used in the Purvis model, is created and told to jump at an appropriate point in time. Once the jumper object impacts the ground level, it is deleted from the simulation.

The Global Module defines and initializes those constants and looping variables that are used throughout the simulation. It includes the method initializeData that initializes the constants used in trajectory propagation. The Main Module imports this method and implements it prior to creating a new instance of a jumper object.

The Calculation Module defines two functional procedures that are used repetitively during trajectory propagation. The first procedure calculates the gravitational effect at a given altitude. The second procedure calculates the density and speed of sound for a given altitude above sea level (ASL).

The Jumper Module contains the bulk of the MODSIM III code. There are two methods written into the Jumper Module: ObjInit and jump. When the Main Module creates a new instance of a jumper object, the ObjInit method is automatically called upon to initialize all the parameters specific to the jumper in its trajectory propagation. The jump method houses the trajectory propagation loop, where trajectory information is continually calculated until ground impact. During this propagation, the jumper maintains all output information, while displaying it at specified time intervals.

Several assumptions are made regarding the physical properties of the paratrooper-parachute system in order to model its aerodynamic properties. The paratrooper object assumes the physical geometry of a cylinder (six feet high and one foot in diameter), with the T-10C assuming the form of a halfsphere. There is also the conical section of the system formed by the risers connecting the paratrooper to the parachute. Lastly, as the parachute inflates, there is added mass to the system due to the air trapped under the parachute canopy. Although joined at a point where the harness meets the paratrooper, we simplify the system by considering it a rigid body; i.e., the joint does not pivot, and the payload has no relative motion with respect to the parachute.

\section{$3.2 \quad$ Vortex Objects}

Since a real vortex is a continuous turbulent area generated by a passing aircraft, we follow Blake's (1996) technique of representing it as discrete points behind the aircraft. Choosing a step size of 100 feet, each vortex is modeled as a data array consisting of position, strength, age, and radius out to a distance of 42,000 feet beyond the generating aircraft. The distance of a threshold swirl velocity from the vortex center is calculated from equations provide by Wright Laboratories (Blake 1996). This distance is the radius of the vortex the model will consider sufficiently close to count as an encounter if a paratrooper glides within it.

The principle method of the vortex object involves the requirement to update the position and strength of each of its 420 discrete representations. This update occurs every $1 / 2$ second of simulated time, and incorporates wind effects and aircraft perturbations. Finally, while each aircraft generates one vortex for each wing, it is the paratrooper objects that contain the method for determining their individual proximity to each vortex.

\section{$3.3 \quad$ C-17 Object}

The C-17 object is the simplest of the four. The main purpose of this object is to generate a vortex characteristic of the C-17 wing, and hold the information necessary for vortex calculations (i.e., wing area, aspect ratio, and wing span). The two main methods in the C-17 object - FlyPosition and greenLight - fly the aircraft and initiate paratrooper exits, respectively. 


\subsection{Control Object}

The control object, TotalVortexObj, combines these objects to simulate airborne operations by (i) managing positional information using three coordinate systems; (ii) modeling separate right and left paratrooper objects; (iii) passing relevant information between the C-17 aircraft objects and the individual paratrooper objects; (iv) initiating the greenLight method by which the paratrooper objects begin jumping; (v) a method for each paratrooper object to calculate its own distance from vortices originating from upstream aircraft; and, (vi) generating the major sources of random influences in the system.

Coordinate Systems. The use of three related coordinate systems is a convenient way of managing relative positional information between the C- 17 aircraft, vortices, and paratrooper objects. The first coordinate system, its origin centered on the lead aircraft of the lead element and continuously moving, is referred to as the Aircraft Coordinate System (ACS). Positive direction is measured aft of the aircraft for the x-position, starboard for the y-position, and above for the z-position. As the stationary system, the Ground Coordinate System (GCS) has its origin at the approach edge of the drop zone. Positive direction is measured in the direction of flight path for the $\mathrm{x}$-position, to the right for the $\mathrm{y}$-position, and above for the z-position. A third coordinate system, the Inertial Coordinate System (ICS), is unique to the paratrooper objects. Its origin is at the point of exit for the paratrooper object and remains stationary from exit to impact. Positive direction is measured along the direction of flight for the $\mathrm{x}$-position, starboard for the y-position, and downward for the z-direction.

Left and Right Paratrooper Objects. The addition of distinct right and left paratrooper objects adds to the resolution of the simulation by modeling the exit of paratroopers from either the right or left rear doors of the C-17. The only difference between a right and a left paratrooper object is its point of origin.

Communication of Information. Communication between the aircraft and the paratrooper objects is essential for several activities that occur in the simulation. The first instance of shared information is in the greenLight method of the C-17 aircraft objects, which starts the stick of paratrooper objects exiting the aircraft. In this method, the C-17 aircraft object passes on its positional information to the paratrooper objects. Once free of the aircraft, the paratrooper objects act independently of other objects; however, information about exit positions, winds, and vortex positions are continuously passed into the paratrooper objects, since this information changes concurrently with the paratrooper objects' trajectories.

Green Light. In airborne operations, aircraft are flying in a specified formation. Green Light is called when the CARP is encountered. The CARP in the simulation is similar to the CARP in Martin and Hypher's (1978) model in that it is the point in airspace where paratrooper objects begin their exit from the aircraft. However, it differs in that it is not calculated from a planned impact point on the $\mathrm{DZ}$, but rather used as a starting point for the simulation. Once Green Light is reached, paratrooper objects begin to exit the aircraft until the last paratrooper object in the stick has departed. Paratrooper objects exit with a constant inter-departure time of 0.5 seconds.

Vortex Polling. Management of positional information allows for each paratrooper object to calculate its position in all three coordinate systems. Relative positioning is used in determining paratrooper object distances from known vortex positions via the polIVortices method. Vector projection is used to find the orthogonal distance between the paratrooper object and the vortex object's core, which we recall is defined as points in space at 100 -foot intervals beginning 100 feet behind its generating aircraft. In order to minimize the number of calculations, the paratrooper object first identifies which of the 100-foot spaced points of the vortex body fall within a certain distance from it. An orthogonal projection to the vortex core is then calculated for each adjacent pair of these nearby points. If this orthogonal distance is within the effective vortex radius (defined by a critical vortex strength, or swirl velocity) then an encounter is recorded (see Figure 1). Major and minor encounters are not distinguished. The paratrooper object makes these calculations for every upstream vortex.

Random Perturbations. Without any form of randomized behavior in the model, the vortex encounter rate and the DZ dispersion could be calculated deterministically. The trajectories of each paratrooper object would exactly mirror the trajectory of any other paratrooper object, thus resulting in either all or no paratrooper-wake vortex encounters. Thus, random variables are incorporated such that the simulation better reflects their true behavior in an airborne operation. Although a variety of elements are candidates for stochastic representation, we limit the number to paratrooper weights, T-10C glide, winds, and aircraft position in formation. In keeping with resolution and capabilities of the Purvis model, these first two elements are easily modeled without changing the fundamental aerodynamic methodology used. (The aircraft and vortex objects handle the variation in wind and aircraft position.) From the weights of paratroopers used in D-bag clearance testing in March 1996, a nor- 


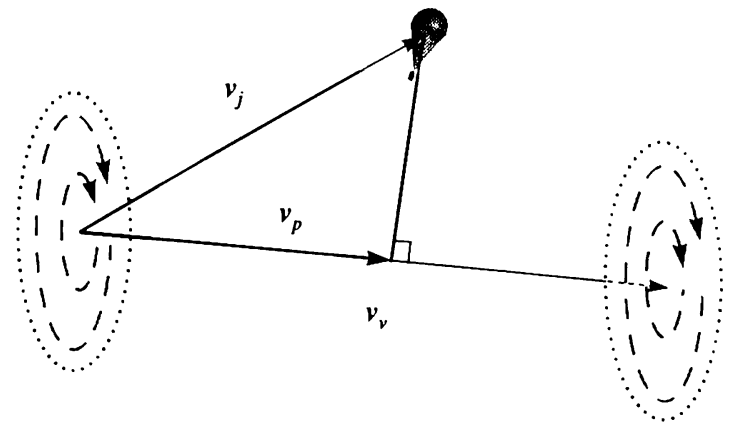

Figure 1: Orthogonal Distance of Paratrooper from Vortex Core

mal distribution was found to adequately model paratrooper body and equipment weight with a mean of 247 pounds and a standard deviation of 24.35 pounds.

A harder problem is the random glide inherent in the $\mathrm{T}-10 \mathrm{C}$ when no wind is present and no oscillation is being experienced. In our approach, when the paratrooper object has reached steady state descent, the onset of glide is modeled from a uniform draw between 0 and 360 degrees for direction, and between 0 to 4 feet-per-second (fps) for velocity. Subsequent changes in glide direction are random - but minor and infrequent - in order to approximate the observed stability of real paratroop trajectories, while the descent rate continually varies between 0 to $4 \mathrm{fps}$ (Watkins 1996).

\subsection{Verification}

For the paratroops objects, multiple runs were made to determine the largest absolute error in their state variables as compared to the state variables of the FORTRAN model. Based on these small errors, we conclude that the Purvis FORTRAN model has been correctly translated into MODSIM III, since the errors are small enough to be attributable to computational differences in the hardware or software environment. Additional verification was accomplished by comparing the relational information of the paratrooper objects; e.g., exit aircraft, the time of exit, the $(x, y, z)$-coordinate of the exit point, airspeed at time of exit, and wake vortices from upstream aircraft, to what would be expected in an actual formation.

\subsection{Validation}

Two basic validation tests were conducted. The first one reproduced a flight test at Edwards AFB, and compared the model's encounter rate with the observed proportion in the actual test. The Edwards test involved two $\mathrm{C}-17 \mathrm{~s}$, one flying directly behind the other 15,000 feet apart, and heading directly into the prevailing wind to minimize any crosswind effects. Mannequins were released from the second aircraft and scored visually by ground observers to count the number of vortex encounters. Assuming each paratrooper has an identical and independent chance of encountering a vortex, the estimated encounter rate for these conditions is $0.1625 \pm .0817$ at a $95 \%$ level of confidence. Our simulation of the Edwards flight gave an estimated encounter of $0.135 \pm .05$, with a subsequent test for equal means at a $95 \%$ confidence level showing that there is no statistical difference between the two estimated rates.

The second validation test plotted the dispersal of paratroopers in the Nijmegen drop zone at $\mathrm{Ft}$. Bragg. The simulation assumed a no-wind condition, varied the lateral separation between two C-17s from a distance of 0 to 500 feet, and the trail distance from 15,000 to 32,000 feet. The resulting dispersal of the simulated ground impact points was consistent with our expectations - the distribution of paratroopers along the length of the drop zone was uniform, while their lateral dispersal followed a bimodal pattern (Figure 2). This pattern clearly shows four distinct groups of paratroopers arranged in this fashion due to (i) aircraft lateral separation of 500 feet, and (ii) separation of the paratroopers within each C-17 into the two sticks exiting from either side of the aircraft.

\section{RESULTS}

Our initial analysis focused on determining the relationship between the element lead separation distance of a formation, and its effect on the paratroopervortex encounter rate. We therefore ran the simulation under two scenarios - calm winds and a 5-knot crosswind - starting at 9,000 foot lead-to-lead separation and extending this distance at 6,000 foot increments up to 33,000 feet (see Figures 3 and 4). Figures 5 and 6 plot the results, from which two fundamental observations become clear. First, as expected, crosswinds affect the encounter rate by significantly reducing the number of encounters. Second, for calm winds the rate of encounter drops significantly as the formation is spread out, with diminishing returns occurring beyond 21,000 feet. 


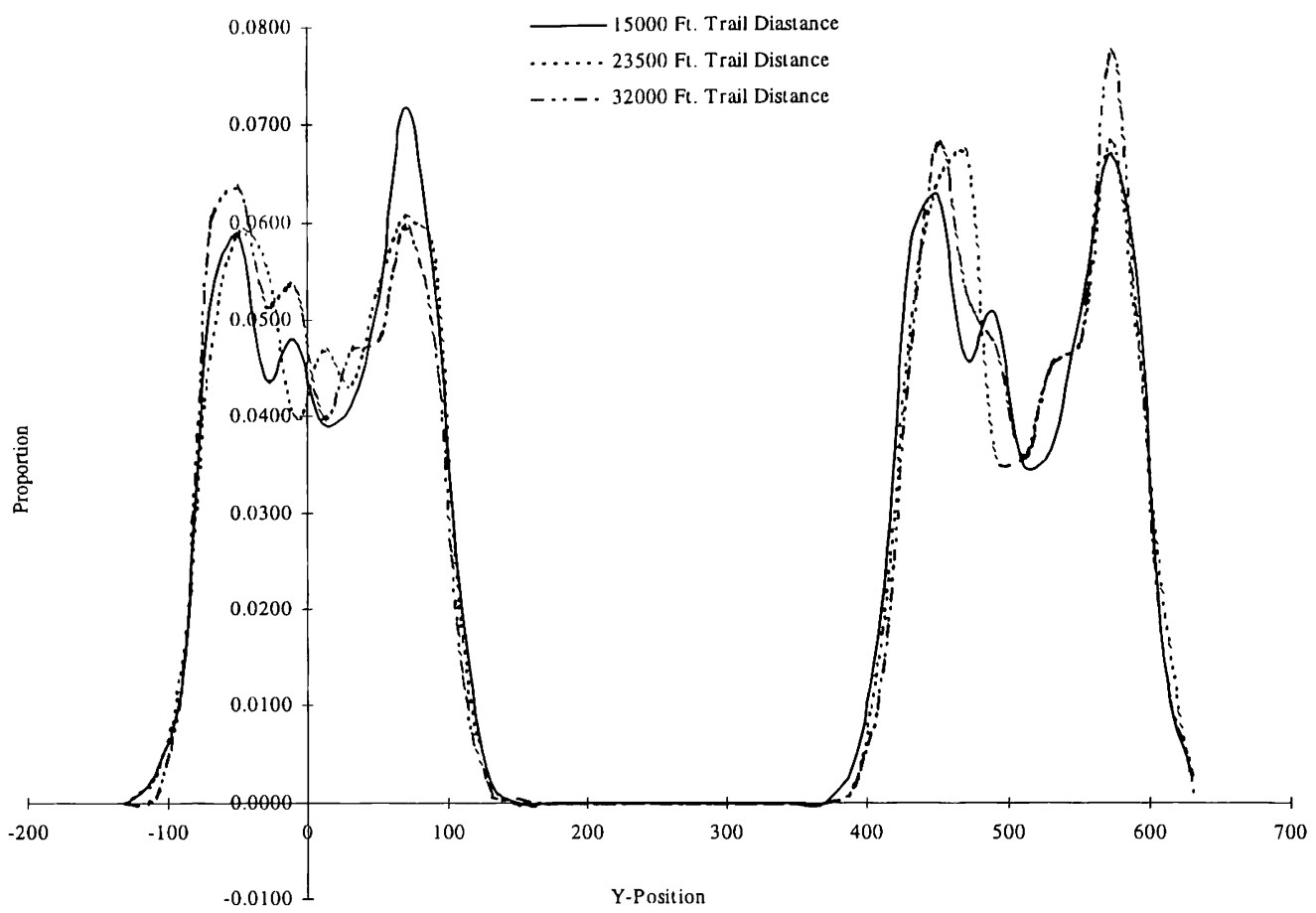

Figure 2: Simulated Paratrooper Dispersion Across Drop Zone with C-17 Lateral Separation of 500 Feet

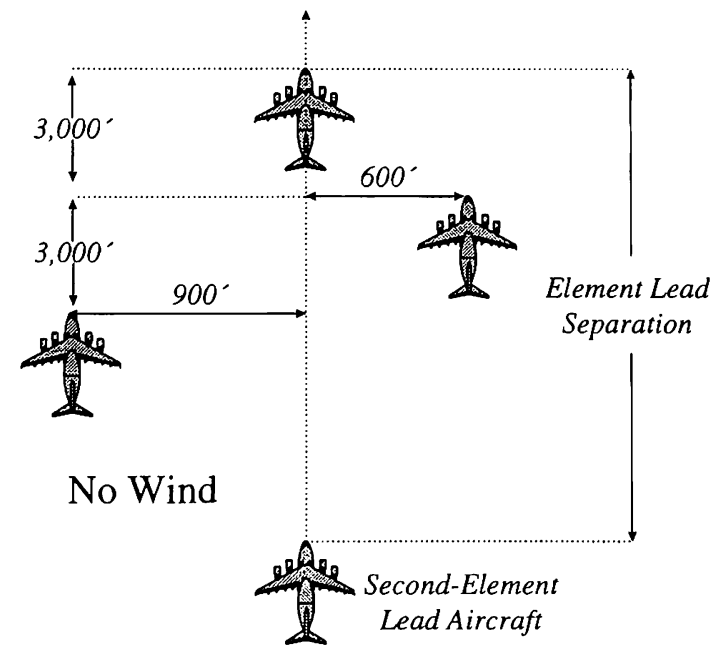

Figure 3: No-Wind Formation $\left(0^{0}\right.$ Drift $)$

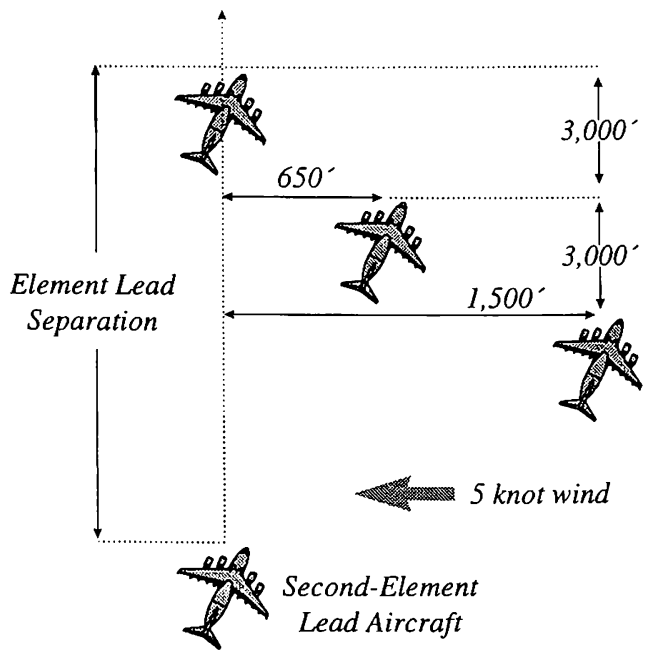

Figure 4: 5-Knot Crosswind Formation ( $3^{0}$ Drift) 


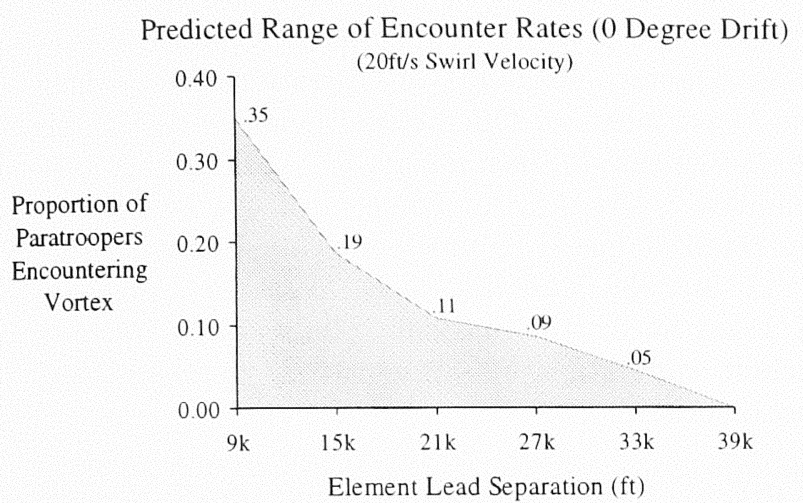

Figure 5: Predicted Range of Paratrooper-Vortex Encounter Rate for No-Wind Formation

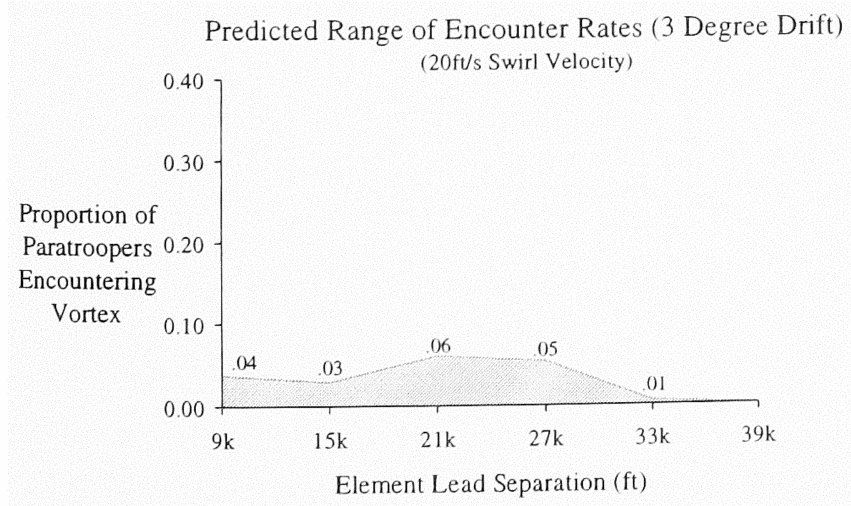

Figure 6: Predicted Range of Paratrooper-Vortex Encounter Rate for 5-Knot Crosswind Formation

\section{CONCLUSIONS}

While the C-17 Airdrop model has provided the joint airborne community with a tool to estimate paratroop-vortex encounter rates, and a mechanism for comparing alternative formations before expensive flight tests, there is still room for improvement.

With respect to modeling the paratroopers, we propose the following. First, the payload is assumed non-intelligent or "unconscious", when in reality paratroopers control the direction of flight under a $\mathrm{T}-10 \mathrm{C}$ canopy. Second, the right and left paratrooper objects exit the C-17 aircraft object at discrete time intervals every 0.5 seconds, although in fact this interdeparture time between them is random. Third, paratrooper objects immediately assume the velocity conditions of the prevailing winds. Fourth, the paratrooper trajectory propagation scheme is not affected by the presence of wake vortices; i.e., paratrooper objects do not interact with the wake vortices, thus causing the impact points to be determined without taking into account the effect of an encounter.

Turning to the aircraft and vortex objects, we suggest the following improvements. First, since vortex strength diminishes in a random fashion, its length is a random variable and should be modeled as such. Second, a more accurate method of modeling wind behavior is needed; currently, the method for simulating this is relatively crude. Finally, how aircraft fly in relation to the lead aircraft needs to be refined.

We conclude with the observation that the objectoriented nature of this model provides many advantages. From a programming perspective it has been easy to modify, scales easily (from single twoship formations to strategic brigade-level airdrops), and delivers a simple framework for expanding the model to include non-personnel offload and other aircraft through the addition of their representative objects. Most interestingly, we found the objectoriented paradigm provides an excellent basis for verification and validation with their real-world counterparts. Since the objects were easy to understand and explain, we found that experts in the areas of airdrop, vortex, and aircraft performance could immediately identify with their portion of the model.

\section{ACKNOWLEDGMENTS}

The authors gratefully acknowledge the C-17 Systems Program Office, Wright-Patterson AFB, OH, for their support of this research. 


\section{REFERENCES}

Benney, Richard. 1996a. 3-DOF Round Parachute System Oscillation Model. Report to C-17 SPO, U.S. Army Natick Research, Development, and Engineering Center, Natick, MA.

Benney, Richard. 1996b. Personal Interview. U.S. Army Natick Research, Development, and Engineering Center, Natick, MA.

Blake, William. 1996. Prediction of Paratrooper Vortex Encounters During Formation Airdrop. Report to C-17 SPO, Wright Laboratory, WrightPatterson AFB, OH.

Doherr, Karl-Friedrich. 1992. Nine-Degree-ofFreedom Simulation of Rotating Parachute System. Journal of Aircraft 29:774-781.

Hannon, S.M., J.A. Thomas, S.W. Henderson, and R. Huffaker. 1995. Windshear, Turbulence, and Wake Vortex Characterization Using Pulsed Solid-State Coherent LIDAR. In Proceedings of the SPIE - The International Society for Optical Engineering 2464:94-102.

Johnson, David J. 1988. Operational Test and Evaluation of the Effects of C-130/C-141B Wake Vortices on the Drop Zone Environment. MAC Project 15-105-86, Scott AFB, IL.

Johnson, David J. and Jon K. Reynolds. 1988. Operational Test and Evaluation of the Effects of C-5 Wake Vortices on the Drop Zone Environment. MAC Project 15-105-86-1, Scott AFB, IL.

Martin, Grant and R. Peter Hyper. 1978. Use of Simulation in the Study of Paradrop. 11th Annual Simulation Symposium, Tampa, FL.

Natick Mobility Directorate. 1996a. Preliminary Proposal for Possible Methods to Determine Acceptable Wake Strength for Personal Parachutes. Report to C-17 SPO, U.S. Army Natick Research, Development, and Engineering Center, Natick, MA.

Natick Mobility Directorate. 1996b. Preliminary Summary Report of Parachute Vortex Interaction Testing with C-141B and (-17A Aircraft. Report to C-17 SPO, U.S. Army Natick Research, Development, and Engineering Center, Natick, MA.

Purvis, J. 1987. Six Degrees of Freedom Flight Simulation Directed Cosine-Euler Axes Method: A Single-Body Parachute Deceleration Program. CCG-Course F12.01, Muenchen, GE.

Tory, C. and R. Ayres. 1977. Computer Model of a Fully Deployable Parachute. Journal of Aircraft 14:675-679.

Wallace, Peter. 1996. Report on the Accuracy of Parachute Delivery of the Container Delivery System. Report to Wright Laboratory, U.S. Army Natick Research, Development, and Engineering
Center, Natick, MA.

Watkins, John. 1996. Personal Correspondence. U.S. Army Natick Research, Development, and Engineering Center, Natick, MA.

\section{AUTHOR BIOGRAPHIES}

T. GLENN BAILEY, Lieutenant Colonel, USAF, is an Assistant Professor of Operations Research in the Department of Operational Sciences at the Air Force Institute of Technology, WPAFB, OH. A 1978 graduate of the U.S. Air Force Academy, Lt Col Bailey received his M.S. in Operations Research from AFIT in 1988, and his Ph.D. from the University of Texas at Austin in 1995. His research interests include simulation, response surface methods, and metaheuristics.

JOSE C. BELANO III, Captain, USAF, received his B.S. in Operations Research from the U.S. Air Force Academy in 1992, and his M.S. in O.R. from the Air Force Institute of Technology in 1997. He is currently assigned as an analyst with the National Security Agency, Ft. Meade, MD.

PHILIP S. BERAN is an Associate Professor of Aeronautical Engineering at the Air Force Institute of Technology, WPAFB, OH. He received his B.S. in Engineering Physics from Cornell University in 1982, and his M.S. and Ph.D. in Aeronautics in 1983 and 1989, respectively, from the California Institute of Technology. Dr. Beran's research interests include computational fluid dynamics, computational aerodynamics, and vortex breakdowns.

JACK M. KLOEBER, Jr., Lieutenant Colonel, USA, is an Assistant Professor of Operations Research in the Department of Operational Sciences at the Air Force Institute of Technology, WPAFB, OH. He received his B.S. and M.S. in Industrial Engineering in 1977 and 1988, respectively, from Lehigh University, and his Ph.D. from Georgia Tech in 1995. His research interests are in the areas of combat modeling, game theory, and simulation.

HANS J. PETRY, Major, USAF, received his B.S. in Engineering Sciences from the U.S. Air Force Academy in 1983, and his M.S. in O.R. from the Air Force Institute of Technology in 1997. A prior C-141B instructor pilot for airdrop and Special Operations Low Level I and II missions, Major Petry is currently assigned as an analyst at Headquarters, Air Mobility Command, Scott AFB, IL. 